\title{
Spin accumulation and decay in magnetic Schottky barriers
}

\author{
Gerrit E. W. Bauer, ${ }^{1}$ Yaroslav Tserkovnyak, ${ }^{2}$ Arne Brataas, ${ }^{3}$ Jun Ren, ${ }^{4} \mathrm{Ke}$ Xia, ${ }^{4}$ Maciej Zwierzycki, ${ }^{5}$ and Paul J. Kelly ${ }^{5}$ \\ ${ }^{1}$ Kavli Institute of NanoScience, Delft University of Technology, Lorentzweg 1, 2628 CJ Delft, The Netherlands \\ ${ }^{2}$ Lyman Laboratory of Physics, Harvard University, Cambridge, Massachusetts 02138, USA \\ ${ }^{3}$ Department of Physics, Norwegian University of Science and Technology, N-7491 Trondheim, Norway \\ ${ }^{4}$ Institute of Physics, CAS, Beijing 10080, People's Republica of China \\ ${ }^{5}$ Faculty of Science and Technology and MESA + Research Institute, University of Twente, 7500 AE Enschede, The Netherlands
}

(Received 27 June 2005; published 5 October 2005)

\begin{abstract}
The theory of charge and spin transport in forward-biased Schottky barriers reveals characteristic and experimentally relevant features. The conductivity mismatch is found to enhance the current-induced spin imbalance in the semiconductor. The GaAs|MnAs interface resistance is obtained from an analysis of the magnetic-field-dependent Kerr rotation experiments by Stephens et al. and compared with first-principles calculations for intrinsic interfaces. With increasing current bias, the interface transparency grows toward the theoretical values, reflecting increasingly efficient Schottky barrier screening.
\end{abstract}

DOI: 10.1103/PhysRevB.72.155304 PACS number(s): 73.30. $+\mathrm{y}$, 72.25.Hg, 72.25.Mk, 78.47.+p

\section{INTRODUCTION}

An obstacle to the direct injection of spins from a ferromagnetic metal (F) into a semiconductor (SC) is the socalled conductivity mismatch. ${ }^{1}$ Paradoxically, this problem is most severe for good electric contact, because most of the applied potential drop is then wasted over the highly resistive semiconductor and very little is left to spin-polarize the current in the magnetically active region. Selection rules at ballistic interfaces are responsible for a large interface spin polarization $^{2,3}$ that allows significant spin accumulation in spite of the mismatch, but even small amounts of disorder have detrimental effects. ${ }^{3}$ Use of ferromagnets with low conductances matched to those of the semiconductor ${ }^{4}$ is another route, but many material problems, such as low critical temperatures for ferromagnetism, have still to be solved. ${ }^{5}$ Spins can be effectively injected into a semiconducting base contact of a three-terminal spin-flip transistor ${ }^{6}$ or by pumping spins into the semiconductor by ferromagnetic resonance, ${ }^{7}$ but none of these theoretical predictions have been confirmed experimentally yet. The spin polarization of the injected current can be increased by tunneling or Schottky barriers, both causing the applied potential to drop in the spinselective region of the sample. ${ }^{1,8}$ This feature has been employed in experiments that divide into two categories. In the first, hot electrons are injected into a metallic magnetic multilayer base in the forward-bias regime. In the "spinvalve transistor" " this is achieved via a Schottky barrier; in "magnetic tunnel transistors," tunneling barriers are used instead. ${ }^{10}$ The second category of experiments concentrates on injecting spins from the ferromagnet into semiconductors by applying a reverse bias, reaching polarizations of $30 \% .{ }^{11}$ Here the spin current is the observable, measured by the circular polarization of the recombination luminescence of the injected electrons with thermalized holes.

Recently, Stephens et al. ${ }^{12}$ investigated in forward-biased Schottky barriers not the hot electrons that traverse a ferromagnetic base as in Refs. 9 and 10, but the cold ones that remain in the semiconductor. A significant bias-dependent spin accumulation in the semiconductor was observed by
Kerr rotation. The interpretation as spin-dependent reflection at the interface was supported by a simple parabolic bandstep potential model. In this paper we present a theoretical analysis based upon an adaptation of magnetoelectronic circuit theory. ${ }^{6,14}$ We find that the conductance mismatch has a beneficial effect on the size of the spin accumulation. Analyzing the Bloch equation that governs the spin accumulation in the presence of an applied magnetic field we find that the experimental results on the dephasing by a magnetic field (Hanle effect) are enhanced by the interface and can be used to extract the $\mathrm{SC} \mid \mathrm{F}$ interface resistance which we compare with first-principle calculations.

\section{SPIN AND CHARGE TRANSPORT AT A BIASED SCHOTTKY BARRIER}

The sample configuration is indicated in Fig. 1. We start with a discussion of an infinite planar Schottky barrier model between a degenerately $n$-doped semiconductor SC and a metallic ferromagnet $\mathrm{F}$ that is kept at low temperatures and biased with an electric particle current $I_{C}$. With increasing forward (positive) bias the semiconductor band edge is lifted

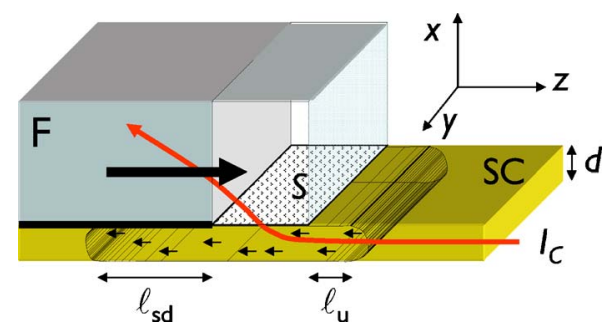

FIG. 1. (Color online) Schematic drawing of the magnetic forward-biased Schottky diode of Stephens et al. (Ref. 12). The particle current $I_{C}$ is injected from the semiconductor film SC of thickness $d$ into the ferromagnet $\mathrm{F}$ through a contact area $S$. The excited spin accumulation diffuses back into the semiconductor over the spin-diffusion length $\ell_{s d}$ without bias and upstream diffusion length $\ell_{u}$ against the bias. The spin accumulation in the semiconductor is plotted for positive interface polarization $p$. 


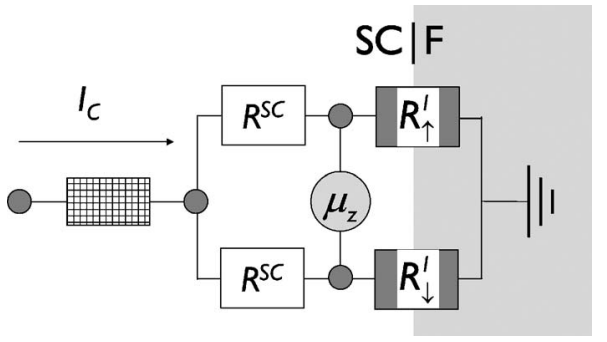

FIG. 2. Magnetoelectronic circuit for the spin accumulation $\mu_{z}$ in a current-biased magnetic Schottky barrier in the absence of a magnetic field.

relative to the ferromagnetic one. The ionized donor atoms are increasingly screened until at a bias close to the Schottky barrier height the semiconductor band edge at the interface comes close to the bulk Fermi energy of the semiconductor $\varepsilon_{F}$. The "flat-band" condition is defined asymptotically at a voltage close to the barrier height where the tunneling current and thus the electric field in the semiconductor start to become significant. Although the theory is valid for arbitrary material combinations we concentrate here on the sample investigated by Stephens et al., in which the GaAs is $n$ doped with densities of $\sim 10^{17} \mathrm{~cm}^{-3}$. With an impurity scattering mean free path of $\sim 30 \mathrm{~nm}$ the semiconductor is safely in the diffuse transport regime. The $I-V$ characteristic in the forward bias shows a band tail close to the Schottky barrier and roughly Ohmic behavior at high bias with a resistance of $300 \Omega$, indicating that the thin semiconductor layer limits the transport. Important parameters are the spin-flip diffusion length of $\ell_{\mathrm{sd}} \simeq 2 \mu \mathrm{m},{ }^{16}$ and a flat-band depletion length of $\sim 20 \mathrm{~nm}$. Any residual band bending can thus be incorporated in the (quantum) interface resistance. The conductance of the high-density metallic ferromagnet MnAs is much higher than that of the semiconductor and disregarded. We concentrate on the dimensionless spin-dependent $(s=\uparrow, \downarrow)$ occupation function $f_{s}(\varepsilon)$ in the semiconductor near the interface at an energy $\varepsilon$ from the band edge. The up-spin direction $\uparrow$ is chosen parallel to the majority spin in the ferromagnet. Close to the flat-band condition the energy of the electrons entering the metal is of the order of the Schottky barrier height which is much larger than the semiconductor Fermi energy. With $-e$ the electron charge, $I_{s}$, the spectral charge current of particles with spin $s$ (in units of $e$ per unit of time) into the metal, is therefore

$$
e I_{s}(\varepsilon)=G_{s}^{I}(\varepsilon) f_{s}(\varepsilon),
$$

where $G_{s}^{I}(\varepsilon)$ the interface conductance at energy $\varepsilon$. The total current of spin $s$ is given integrating over energy

$$
I_{s}=\int I_{s}(\varepsilon) d \varepsilon
$$

We assume a particle current bias of $I_{C}=I_{\uparrow}+I_{\downarrow}$ and introduce the spin current $I_{z}=I_{\uparrow}-I_{\downarrow}$. We assume in the following that energy relaxation is fast, such that the distribution function at the interface is thermalized with nonequilibrium chemical potentials $\mu_{s}$. At low temperatures, assuming local charge neutrality $\mu_{\uparrow}+\mu_{\downarrow}=0$ and an interface conductance that does not vary rapidly on the scale of $\mu_{s}$ :

$$
\begin{gathered}
e I_{z}=\int_{0}^{\varepsilon_{F}+\mu_{\uparrow}} G_{\uparrow}^{I}(\varepsilon) d \varepsilon-\int_{0}^{\varepsilon_{F}+\mu_{\downarrow}} G_{\downarrow}^{I}(\varepsilon) d \varepsilon \\
=e I_{z}^{(0)}+\frac{\mu_{z}}{2} G^{I}\left(\varepsilon_{F}\right), \\
e I_{C}=\int_{0}^{\varepsilon_{F}+\mu_{\uparrow}} G_{\uparrow}^{I}(\varepsilon) d \varepsilon+\int_{0}^{\varepsilon_{F}+\mu_{\downarrow}} G_{\downarrow}^{I}(\varepsilon) d \varepsilon \\
=e I_{C}^{(0)}+\frac{\mu_{z}}{2} p\left(\varepsilon_{F}\right) G^{I}\left(\varepsilon_{F}\right)
\end{gathered}
$$

where $G^{I}=G_{\uparrow}^{I}+G_{\downarrow}^{I}, p=\left(G_{\uparrow}^{I}-G_{\downarrow}^{I}\right) / G^{I}, \mu_{z}=\mu_{\uparrow}-\mu_{\downarrow}$ is the spin accumulation at the interface, and

$$
\begin{gathered}
e I_{z}^{(0)}=\int_{0}^{\varepsilon_{F}} p(\varepsilon) G^{I}(\varepsilon) d \varepsilon, \\
e I_{C}^{(0)}=\int_{0}^{\varepsilon_{F}} G^{I}(\varepsilon) d \varepsilon .
\end{gathered}
$$

We then arrive at the effective circuit in Fig. 2, according to which the spin current $I_{z}=I_{\uparrow}-I_{\downarrow}$ that flows from the semiconductor bulk to the interface reads

$$
e I_{z}=\frac{e I_{z}^{(0)}}{1+G^{I} /\left(2 G^{S C}\right)}
$$

where $G^{S C}$ is the conductance of the magnetically active region of the semiconductor that is discussed in the next section in more detail. The sign of the spin accumulation at the interface

$$
\mu_{z}=-\frac{e I_{z}}{G^{S C}}
$$

is opposite to that of $I_{z}$. A low conductance $G^{S C} \rightarrow 0$ suppresses the spin current, ${ }^{1}$ but not the spin accumulation. By reversing $I_{C}$ and keeping in mind that the interface conductance is in general much smaller and less bias dependent, similar equations hold as well for reversed-bias Schottky barriers. As mentioned above, most experiments on reverse-bias junctions focus on the spin current. The conductance mismatch problem is reflected in Eq. (9), where a small semiconductor conductance is seen to suppress the spin current. In Refs. 1 and 8 it was pointed out that a significantly polarized spin current can only be achieved when the reverse-bias Schottky barrier conductance is sufficiently small. However, in this case the spin accumulation $\mu_{z}$ is suppressed according to Eq. (10), which explains why Stephens et al. ${ }^{12}$ detected spin accumulation with a forward bias only.

\section{SPIN DRIFT IN SEMICONDUCTORS}

The magnetically active region of an unbiased semiconductor is limited by the spin-flip diffusion length $\ell_{\text {sd }}$ $=\sqrt{D^{S C} \tau_{\mathrm{s}}}$, where $D^{\mathrm{SC}}$ is the diffusion coefficient and $\tau_{\mathrm{sf}}$ the 
spin-flip diffusion time. Yu and Flatté ${ }^{17}$ have shown that in low-density conductors, such as doped semiconductors, the density dependence of the mobility significantly affects the transport properties by an electric-field-dependent drift term. Here we derive the electric-field-dependent resistance of the magnetically active region of the semiconductor.

Assuming a spin-dependent conductivity $\sigma_{s}$ that depends on the the spin density $n_{s}$ like

$$
\sigma_{s}^{\mathrm{SC}}=\sigma_{0}^{\mathrm{SC}}+\left(n_{s}-n_{0}\right) \nu^{\mathrm{SC}}
$$

where $\sigma_{0}^{\mathrm{SC}}$ is the linear response conductivity and $\nu^{\mathrm{SC}}$ a constant mobility, Yu and Flatté ${ }^{17}$ derived the diffusion equation for the spin density $n_{z}=n_{\uparrow}-n_{\downarrow}$ in the degenerate limit

$$
\frac{\partial^{2}}{\partial x^{2}} n_{z}(x)+\frac{\nu^{\mathrm{SC}} E}{D^{\mathrm{SC}}} \frac{\partial}{\partial x} n_{z}(x)=\frac{n_{z}(x)}{\left(\ell_{s d}\right)^{2}},
$$

where under the condition of local charge neutrality

$$
n_{s}(x)=e \mathcal{N}^{\mathrm{SC}}\left(\varepsilon_{F}\right)\left[\mu_{s}+e V(x)\right]=-n_{-s}=n_{z} / 2,
$$

and $V(x)$ is the local voltage, $E=-\partial V / \partial x$ the electric field, and $\mathcal{N}\left(\varepsilon_{F}\right)$ the density of states at the Fermi energy. The solution of the diffusion equation reads ${ }^{17}$

$$
n_{z}(x)=A_{1} e^{x / \ell_{u}}+A_{2} e^{-x / \ell_{d}}
$$

in terms of the upstream $\ell_{u}$ (against the current) and downstream $\ell_{d}$ (with the current) diffusion lengths

$$
\frac{\ell_{u, d}}{\ell_{s d}}=\sqrt{1+\left(\frac{E \nu^{\mathrm{SC}} \ell_{s d}}{2 D^{\mathrm{SC}}}\right)^{2}} \pm \frac{E \nu^{\mathrm{SC}} \ell_{s d}}{2 D^{\mathrm{SC}}}
$$

with $\ell_{u} \leqslant \ell_{s d} \leqslant \ell_{d}$. The magnetically active region of the semiconductor is therefore determined by the upstream spin-diffusion length $\ell_{u}=\ell_{s d}\left(\sqrt{1+X^{2}}-X\right)$, where $X$ $=3 e I_{C} /\left(8 G_{0}^{S C} \varepsilon_{F}\right)$ is a measure of the potential drop induced by the current over the (zero-bias) spin diffusion length $\ell_{s d}$ in terms of the linear bulk conductance $G_{0}^{S C}=S \sigma_{0}^{S C} / \ell_{s d}$ of a semiconductor cube with area $S$ and thickness $\ell_{s d}$.

We can solve the diffusion equation for the spin current and spin accumulation for our situation assuming conservation of charge and spin currents at the boundary with the ferromagnet:

$$
\begin{aligned}
& e I_{C}=e I_{C}^{(0)}+\frac{\mu_{\uparrow}-\mu_{\downarrow}}{2}\left(G_{\uparrow}^{I}-G_{\downarrow}^{I}\right), \\
& e I_{z}=e I_{z}^{(0)}+\frac{\mu_{\uparrow}-\mu_{\downarrow}}{2}\left(G_{\uparrow}^{I}+G_{\downarrow}^{I}\right),
\end{aligned}
$$

with the results that

$$
e I_{z}(0)=\frac{e I_{z}^{(0)}}{1+G^{I} \ell_{s d}^{2} /\left(2 \sigma_{0}^{S C} \ell_{u}\right)},
$$

and at the semiconductor side of the interface

$$
\mu_{z}(0)=-\frac{e I_{z}(0) \ell_{s d}^{2}}{2 \sigma_{0}^{S C} \ell_{u}} .
$$

The results are consistent with Eqs. (9) and (10) for the resistor model, allowing us to express the bias-dependent semiconductor conductance

$$
G^{S C}=G_{0}^{S C} \frac{\ell_{u}}{\ell_{s d}}
$$

In spite of the reduced upstream spin-diffusion length, the conductance of the spin-coherent region is decreased compared to the zero-bias limit and not increased by a factor $\ell_{s d} / \ell_{u}$ as might be expected naively.

\section{INTERFACE-ENHANCED HANLE EFFECT}

We now turn to the spin accumulation in the presence of a variable in-plane magnetic field, taking the magnetization of $\mathrm{F}$ to be parallel to the $z$ direction and the magnetic field $B$ in the $y$ direction. The magnetic-field-induced noncollinearity of spin accumulation and magnetization creates a spintransfer torque $\mathrm{e}^{13}$ on the ferromagnet, thus opening new decay channels ${ }^{14}$ proportional to the spin-mixing conductance $G_{\uparrow \downarrow}^{I}$ at the Fermi energy. ${ }^{6}$ The Bloch equation for the spin accumulation $\langle\mu|=\left(\mu_{x}, \mu_{y}, \mu_{z}\right)$ can be written

$$
-T^{I} \frac{d|\mu\rangle}{d t}=\Gamma|\mu\rangle+\frac{2 e\left|I_{z}\right\rangle}{G_{I}},
$$

where $T^{I}=2 e^{2} \mathcal{D} / G^{I}$ is the interface spin-relaxation time in terms of the (single-spin) semiconductor energy density of states $\mathcal{D}$ in the magnetically active volume.

$$
\boldsymbol{\Gamma}=\left(\begin{array}{ccc}
\eta_{r}+\xi & \eta_{i} & T^{I} \omega \\
-\eta_{i} & \eta_{r}+\xi & 0 \\
-T^{I} \omega & 0 & 1+\xi
\end{array}\right)
$$

where $\eta_{r}=2 \operatorname{Re} G_{\uparrow \downarrow}^{I} / G^{I}, \eta_{i}=2 \operatorname{Im} G_{\uparrow \downarrow}^{I} / G^{I}, \quad \xi=2 G^{S C} / G^{I}$, and the Larmor frequency $\omega=g_{e} \mu_{B} B / \hbar$ in terms of the $g$ factor $g_{e}$ and the Bohr magneton $\mu_{B}$. Equation (20) holds when the relaxation rate of the electron orbital degrees of freedom is sufficiently larger than $\omega$. The source term is the current bias applied to the semiconductor. $\left\langle I_{z}\right|=\left(0,0, I_{z}^{(0)}\right)$. The stationarystate solution for the Bloch equation, $|\mu\rangle=-\Gamma^{-1} 2 e\left|I_{z}\right\rangle / G_{I}$, is easily obtained analytically. The spin accumulation at the interface reads

$$
\langle\mu|=\frac{\left(\left(\eta_{r}+\xi\right) T^{I} \omega, \eta_{i} T^{I} \omega,-\left(\eta_{r}+\xi\right)^{2}-\eta_{i}^{2}\right)}{\left[\left(\eta_{r}+\xi\right)^{2}+\eta_{i}^{2}\right](1+\xi)+\left(\eta_{r}+\xi\right)\left(\omega T^{I}\right)^{2}} \frac{2 e I_{z}^{(0)}}{G^{I}} .
$$

Stephens et al. ${ }^{12}$ found the component of the spin accumulation normal to the interface $\mu_{x}$ well represented by a Lorentzian

$$
\mu_{x}=\frac{A \omega}{\omega^{2}+\tau^{-2}} .
$$

This form also follows from our rate equations with

$$
\left(\frac{T^{I}}{\tau}\right)^{2}=\left[\left(\eta_{r}+\xi\right)^{2}+\eta_{i}^{2}\right] \frac{1+\xi}{\eta_{r}+\xi}
$$

and $A=2 e I_{z}^{(0)} /\left(G^{I} T^{I}\right)$. In the limit of a highly resistive semiconductor, $\xi \ll 1$, and taking $\eta_{i}=0, \eta_{r}=1$, we find that $\tau \rightarrow T^{I}$ and $A T \rightarrow \mu_{z}(\omega=0)$, i.e., the zero-field spin accumulation. It is therefore possible to obtain information about the 


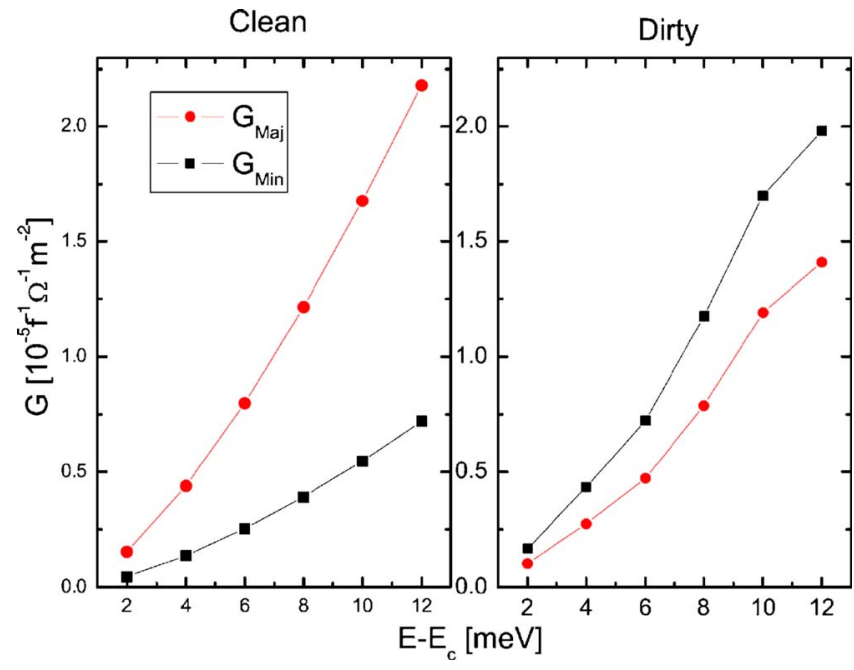

FIG. 3. (Color online) Intrinsic conductance of a specular (left) and disordered (right) zinc-blende $\mathrm{MnAs} / \mathrm{GaAs}$ (100) interface at flat-band conditions as a function of the Fermi energy relative to the GaAs conductance band edge as calculated from first principles.

interface conductance from the experimental spin dephasing time.

\section{BAND STRUCTURE CALCULATIONS}

The MnAs $\mid$ GaAs systems has been studied intensively, ${ }^{15}$ but not much is known about the electronic transport properties. Epstein et al. ${ }^{18}$ reported that the conductance polarization is opposite to the magnetization direction, i.e., $p<0$. We compute MnAs $\mid \mathrm{GaAs}(100)$ interface conductances $G^{I}(\varepsilon)$ for a hypothetical ${ }^{19}$ zinc-blende structure by scattering matrix calculations with a first-principles tight-binding basis, ${ }^{3}$ assuming flat-band conditions with a Schottky barrier height of $0.8 \mathrm{eV}$. We find large differences between clean, and on a monolayer scale, alloy-disordered interfaces, e.g., the interface polarization changes sign when the interface becomes increasingly dirty (see Fig. 3). The negative polarization found in Ref. 18 appears to be consistent with nonideal interfaces, but see Ref. 20. These features are quite similar to results for Fe|InAs that does not have a Schottky barrier. ${ }^{3} \mathrm{We}$ also note that in the regime considered here we calculate an $\eta_{r} \simeq 1$ in all cases and $\eta_{i} \simeq 0(0.35)$ for clean (disordered) interfaces (Fig. 4), both only weakly dependent on the Fermi energy. When parametrizing the estimated interface conductance in terms of the SC Sharvin conductance

$$
\frac{G_{S h}\left(\varepsilon_{F}\right)}{S}=\frac{2 e^{2}}{h} \frac{2 m^{*} \varepsilon_{F}}{4 \pi \hbar^{2}}
$$

times a transparency parameter $\kappa$ we find that (at $\left.\varepsilon_{F}=12 \mathrm{meV}\right) \quad \kappa=0.27$ for clean and dirty interfaces. An energy-averaged interface transparency is accessed by the electrical current. Disregarding the small term proportional to $\mu_{z}$ in Eq. (5), the charge current in the flat-band regime according to the first-principles conductances and a contact area of $250 \mu \mathrm{m}^{2}$ should be $I_{C} \simeq 25 \mathrm{~mA}$, which corresponds to an average transparency $\kappa_{a v}=0.13$.

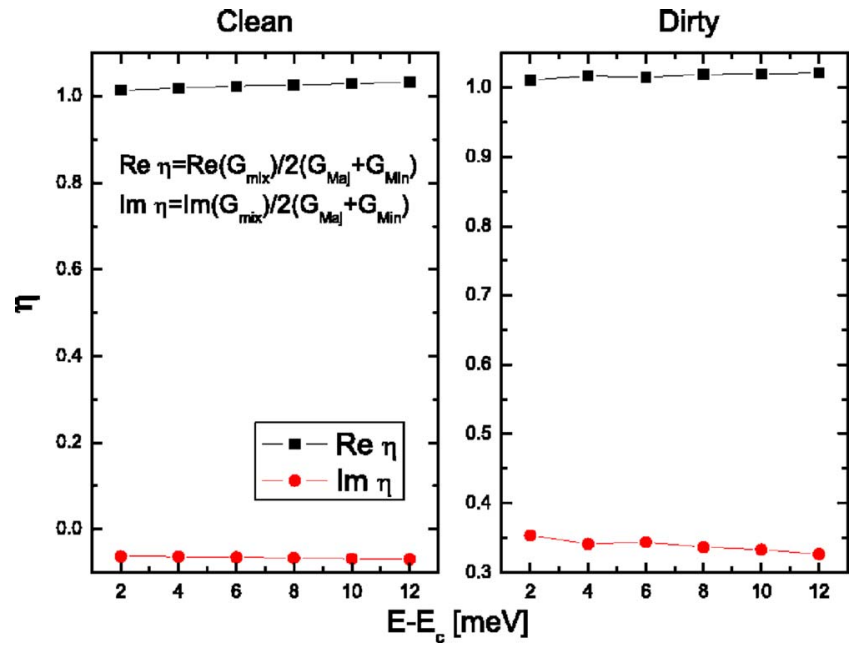

FIG. 4. (Color online) Relative real and imaginary parts of the spin-mixing conductances of a specular (left) and disordered (right) zinc-blende MnAs/GaAs (100) interface at flat-band conditions as a function of Fermi energy relative to the GaAs conductance band edge as calculated from first principles.

\section{EXPERIMENTS}

In order to make contact with Stephens et al. ${ }^{12}$ the above results for the planar junction have to be adapted to the experimental geometry in Fig. 1. For GaAs with doping density $n=10^{17} \mathrm{~cm}^{-3}$, we take a mobility $3000 \mathrm{~cm}^{2} / \mathrm{V} \mathrm{s}$, an effective mass $m^{*}=0.067 m_{e}$, and a spin-flip diffusion length ${ }^{16}$ $\ell_{s d} \simeq 2 \mu \mathrm{m}$, that is significantly larger than the film thickness $d^{S C}=0.5 \mu \mathrm{m}$. The measured excess spin-dephasing rates $\tau^{-1}$ at applied currents $I_{C}$ are listed in Table I. Close to the interface, the upstream spin-flip diffusion length is not significantly reduced, so not only is the whole $\left(\simeq 5 \times 50 \mu \mathrm{m}^{2}\right)$ area under the conducting contact spin coherent at all currents, but also strips on both sides with widths of the order of $\ell_{s d}$ and $\ell_{u}$, respectively. The drift effect on the available density of states is small, but it significantly affects the resistance of the spin-coherent region. Due to the thin layer thickness of the GaAs, lateral spin diffusion may be disregarded. The results in the table are obtained assuming that $\eta_{i}=0, \eta_{r}=1$.

The experimental longevity of spins is striking and can only be explained by a reduced interface conductance. Any spin-flip process disregarded here, caused, e.g., by heating ${ }^{16}$ due to high currents, would correspond to even smaller trans-

TABLE I. Experimental results for the spin dephasing rate $1 / \tau$ at selected current bias $I_{C}$ from Ref. 12 and the estimates of device parameters according to the discussion in the text. $R^{S C}$ and $S R^{I}$ are the SC bulk resistance of the magnetically active region and the interface resistance for the given bias. $\kappa$ is the transparency parameter that measures the interface conductance in units of the SC Sharvin conductance.

\begin{tabular}{ccccc}
\hline \hline$I_{C}(\mathrm{~mA})$ & $1 / \tau\left(\mathrm{ns}^{-1}\right)$ & $R^{S C}(\Omega)$ & $S R^{I}\left(\frac{\mathrm{f} \Omega \mathrm{m}^{2}}{10^{-5}}\right)$ & $\kappa$ \\
\hline 0.3 & 0.25 & 43 & 25 & 0.004 \\
1.1 & 1.2 & 63 & 5.2 & 0.014 \\
2.7 & 6 & 111 & 1.1 & 0.074 \\
\hline \hline
\end{tabular}


parencies. The small $\kappa$ at small bias reflects the residual Schottky barrier that is not yet completely screened. At higher bias these remnants should disappear and the spindephasing time should be governed by the intrinsic interface. At higher bias the interface conductances deduced from the experiments grow to about one-third of the intrinsic firstprinciples results. For the maximal experimental current bias $I_{C}=2.7 \mathrm{~mA}$, we get $\kappa_{a v}=0.014$ which is smaller than that at the Fermi energy obtained from the Hanle effect (Table I). The same situation holds for smaller currents. This can be explained by an energy-dependent $\kappa$ that decreases strongly when approaching the band edge. These remaining puzzles might be related to the measured ${ }^{12}$ spatial inhomogeneity of the current-induced spin accumulation and thus interface conductance.

Stephens et al. ${ }^{12}$ estimate the spin accumulation to be $10 \%$ of the Fermi energy from nuclear polarization data compared to an estimate of $\sim 15 \%$ based on the firstprinciples results for disordered interfaces. The spin accumulation is found to saturate and even decrease again with large $I_{C}$. A probable reason is a reduced spin-flip diffusion length, either by heating or by a large drift contribution at higher bias.

\section{DISCUSSION AND CONCLUSIONS}

In summary, we demonstrate how a transport property, the semiconductor|ferromagnet interface conductance, can be measured optically on an absolute scale. The conductance mismatch is found to favor spin injection into semiconductors in forward-biased magnetic Schottky barriers. When measuring the spin accumulation by Faraday or Kerr rotation experiments the apparent spin-flip dephasing time is strongly modified by the presence of the ferromagnetic interface. The noncollinearity between spin accumulation and the ferromagnetic order parameter opens a spin-decay channel by the spin-transfer torque. The corresponding time constant $T^{I}$ represents dominantly a spin lifetime $T_{1}$ rather than a dephasing time $T_{2}^{*}$. At low bias the interface-mediated spin decay is much weaker in the experiments ${ }^{12}$ than expected from intrinsic $\mathrm{SC} \mid \mathrm{F}$ interfaces. At higher bias the agreement becomes better, indicating that the interface approaches (but does not reach) the Ohmic limit as calculated from first principles. Experiments that determine the spin accumulation on an absolute scale would be of great help to refine the present analysis. The observed negative polarization ${ }^{12,18}$ can be explained by the band structure calculations. A systematic study as a function of semiconductor thickness could shed more light on the spin decoherence in the nonlinear transport regime.

\section{ACKNOWLEDGMENTS}

We thank Georg Schmidt, David Awschalom, and Jason Stephens for helpful discussions. This work has been supported by the FOM Foundation, the EU Commission FP6 NMP-3 project 505587-1 "SFINX", and NFSC Grant No. 90303014.
${ }^{1}$ G. Schmidt, D. Ferrand, L. W. Molenkamp, A. T. Filip, and B. J. van Wees, Phys. Rev. B 62, R4790 (2000).

${ }^{2}$ O. Wunnicke, Ph. Mavropoulos, R. Zeller, P. H. Dederichs, and D. Grundler, Phys. Rev. B 65, 241306(R) (2002).

${ }^{3}$ M. Zwierzycki, K. Xia, P. J. Kelly, G. E. W. Bauer, and I. Turek, Phys. Rev. B 67, 092401 (2003).

${ }^{4}$ R. Fiederling, M. Keim, G. Reuscher, W. Ossau, G. Schmidt, A. Waag, and L. W. Molenkamp, Nature (London) 402, 787 (1999); Y. Ohno, D. K. Young, B. Beschoten, F. Matsukura, H. Ohno, and D. D. Awschalom, ibid. 402, 790 (1999).

${ }^{5}$ H. Ohno, in Physics of Semiconductors 2002 (Proceedings of the 26th International Conference on the Physics of Semiconductors, Edinburgh, 2002), IOP Conf. Proc. No. 171, A. R. Long andJ. H. Davis\&, eds (IO: Conference Series No. 171, IOP Publishing, Bristol, 2003).

${ }^{6}$ A. Brataas, Yu. V. Nazarov, and G. E. W. Bauer, Phys. Rev. Lett. 84, 2481 (2000); Eur. Phys. J. B 22, 99 (2001).

${ }^{7}$ A. Brataas, Y. Tserkovnyak, G. E. W. Bauer, and B. I. Halperin, Phys. Rev. B 66, 060404(R) (2002).

${ }^{8}$ E. I. Rashba, Phys. Rev. B 62, R16267 (2000);A. Fert and H. Jaffres, ibid. 64, 184420 (2001).

${ }^{9}$ D. J. Monsma, J. C. Lodder, Th. J. A. Popma, and B. Dieny, Phys. Rev. Lett. 74, 5260 (1995);O. M. J. van’t Erve, R. Vlutters, P. S. Anil Kumar, S. D. Kim, F. M. Postma, R. Jansen, and J. C. Lodder, Appl. Phys. Lett. 80, 3787 (2002).

${ }^{10}$ S. van Dijken, X. Jiang, and S. P. Parkin, Appl. Phys. Lett. 83, 951 (2003);I. Appelbaum, K. J. Russell, D. J. Monsma, V.
Narayanamurti, C. M. Marcus, M. P. Hanson, and A. C. Gossard; ibid. 83, 4571 (2003)

${ }^{11}$ O. M. J. van’t Erve, G. Kioseoglu, A. T. Hanbicki, C. H. Li, B. T. Jonker, R. Mallory, M. Yasar, and A. Petrou, Appl. Phys. Lett. 84, 4334 (2004), and references therein.

${ }^{12}$ J. Stephens, J. Berezovsky, J. P. McGuire, L. J. Sham, A. C. Gossard, and D. D. Awschalom, Phys. Rev. Lett. 93, 097602 (2004).

${ }^{13}$ J. C. Slonczewski, J. Magn. Magn. Mater. 159, L1 (1996).

${ }^{14}$ G. E. W. Bauer, A. Brataas, Y. Tserkovnyak, B. I. Halperin, M. Zwierzycki, and P. J. Kelly, Phys. Rev. Lett. 92, 126601 (2004);M. Zaffalon and B. J. van Wees, Phys. Rev. B 71, 125401 (2005).

${ }^{15}$ M. Tanaka, Semicond. Sci. Technol. 17, 327 (2002).

${ }^{16}$ J. M. Kikkawa and D. D. Awschalom, Phys. Rev. Lett. 80, 4313 (1998).

${ }^{17}$ Z. G. Yu and M. E. Flatté, Phys. Rev. B 66, 235302 (2002).

${ }^{18}$ R. J. Epstein, I. Malajovich, R. K. Kawakami, Y. Chye, M. Hanson, P. M. Petroff, A. C. Gossard, and D. D. Awschalom, Phys. Rev. B 65, 121202(R) (2002).

${ }^{19}$ S. Sanvito and N. A. Hill, Phys. Rev. B 62, 15553 (2000).

${ }^{20}$ Preliminary results on a geometry-optimized hexagonal $\alpha$-MnAs $\mid$ GaAs interface show interesting differences, such as a negative polarization for specular interfaces and a stronger dependence of $\kappa$ on disorder morphology [P.-X. Xu et al. (unpublished)]. 